\title{
TEXTURES FOR NEUTRINO MASS MATRICES IN GAUGE THEORIES
}

\author{
S. Lola ${ }^{a}$ and J. D. Vergados $\rrbracket^{b}$ \\ ${ }^{a}$ CERN Theory Division, CH-1211, Switzerland \\ $b$ Theoretical Physics Division, Ioannina University, Ioannina, Greece
}

\begin{abstract}
We review previous work on neutrino mass textures in gauge theories. Such textures may arise as a result of flavour symmetries. In a given theory, it is possible to give a classification of heavy Majorana neutrino mass matrices, assuming the Dirac masses of the neutrinos to be of the same form as the ones of the up-quarks. Heavy Majorana neutrino mass matrices leading to large neutrino mixing, are tabulated as an example. Such solutions are now of interest, in the light of the Super-Kamiokande data.
\end{abstract}

\section{Introduction}

Although the Standard Model successfully describes the strong and electroweak phenomena, there are still various open questions, mainly related to the origin of fermion masses and mixing angles. An obvious way to explain the observed hierarchies that we see at low energies, would be to assume that some symmetry (additional to that of the Standard Model) is responsible for these patterns. An indication that such additional symmetries are present, has been the observation that the fermion mixing angles and masses have values consistent with the appearance of "texture" zeros in the mass matrices [1, 2].

On the other hand, neutrino data from several experiments (including recent results from SuperKamiokande[3]) seem to require certain mixings between various types of massive neutrinos. For these, similar hierarchies are to be expected. In the framework of the Standard Model, neutrinos are massless, since both Dirac and Majorana masses are forbidden by the particle content and the symmetries of the theory: A Dirac mass term $m\left(\overline{\nu_{L}} \nu_{R}+\overline{\nu_{R}} \nu_{L}\right)$ does not arise, due to the absence of right handed neutrinos. In addition, although neutrinos do not carry charge, a Majorana term $m \nu_{L}^{T} \sigma_{2} \nu_{L}$ which would violate lepton number is also forbidden because it is not $S U(2)$ invariant (this term transforms like an $I=1$ object under $S U(2)$ ). However, in GUTS, the symmetries of the theory allow the presence of such terms. Moreover, it is possible to add (at least) one right handed particle in the models, thus raising the possibility of a Dirac mass term as well. If

\footnotetext{
${ }^{1}$ Lecture given by J.D.Vergados, Erice 1997, published in Progress in Particle and Nuclear Physics 40 (1998) 71.
} 
both a Dirac mass and a Majorana mass (for the isosinglet) neutrino are present, the See-Saw mechanism may explain the lightness of neutrinos relative to the charged fermions.

The most general neutrino mass matrix for three flavours for both the isodoublet and isosinglet neutrinos in the current eigenstate basis

$$
\begin{aligned}
& \chi_{R}^{0}=\left(\nu_{R}^{0 c}, \nu_{R}^{0}\right) \\
& \bar{\chi}_{L}^{0}=\left(\bar{\nu}_{L}^{0}, \bar{\nu}_{L}^{0 c}\right)
\end{aligned}
$$

takes the form

$$
M=\left(\begin{array}{cc}
M_{L} & D \\
D^{T} & M_{R}
\end{array}\right)
$$

where $M$ a complex symmetric matrix. The submatrix $M_{L}$ describes the masses arising in the left-handed sector, $D$ is the usual Dirac mass matrix and $M_{R}$ contains the entries in the righthanded isosinglet sector. The mass eigenstates are given by $\nu_{R}=U^{R} \chi_{R}^{0}, \nu_{L}=U^{L} \chi_{L}^{0}, U^{R}$ being a unitary matrix that diagonalizes $M$ from the right and $U^{L}=\left(U^{R}\right)^{*} \times \exp (i \lambda) \cdot \exp (i \lambda)$ is a diagonal matrix of phases(CP-parities of the eigenstates. For a real symmetric matrix the signs of the corresponding eigenvalues) [4]. One rarely deals with the $6 \times 6$ matrix. In practice one deals with $M_{R}$ amd $m_{\nu}^{e f f}$ ('see-saw' mechanism,see sect.2)

Massive neutrinos have important implications for both particle physics and cosmology. The models which admit neutrinos with the desired properties are usually severely constrained, therefore any experimental evidence about neutrino masses and mixing will be a powerful probe of physics beyond the standard model.

There are however experimental limits on the masses and mixing of neutrinos.

- Searches for neutrino masses in $\beta$ decays give the limit $m_{\nu_{e}} \leq 7.3 \mathrm{eV}$.

- Constraints on the mass of $\nu_{\mu}$ are derived via the decay process

$$
\pi^{+} \rightarrow \mu^{+}+\nu_{\mu}
$$

Then, a bound $m_{\nu_{\mu}} \leq 170 \mathrm{keV}$ is derived.

- In a similar manner, the $\nu_{\tau}$ is constrained by the process

$$
\tau^{-} \rightarrow 2 \pi^{+} 3 \pi^{-} \pi^{0} \nu_{\tau}
$$

to be $\nu_{\tau} \leq 24 \mathrm{MeV}$.

- An important constraint on Majorana neutrino masses for the electron neutrino, arises from searches for neutrinoless double $\beta$ decay [4]

$$
d+d \rightarrow u+u+e+e
$$

This process involves violation of lepton number by two units. The most popular scenario involves intermediate Majorana neutrinos (where the particle is the same as its antiparticle). It can also occur via many other mechanisms(intermediate exotic scalars, R-parity violating interactions 
etc).These other processes , however, necessarily imply that the neutrino is a majorana particle.So observation of this process will show that the neutrinos are majorana particles. No signal has been seen so far, which constrains the magnitude of a possible mass term to be less than a few $\mathrm{eV}$.

- If neutrinos are massive, the current eigenstates, $\nu_{i}$, can be written as superpositions of the physical particles, $\nu_{a}$

$$
\nu_{i}(t)=\sum_{a} U_{i a} \nu_{a}
$$

where $\nu_{i}$ are the current and $\nu_{a}$ the mass eigenstates respectively and $U_{i a}$ is a unitary matrix which diagonalizes the neutrino mass matrix. The evolution equation of a purely current eigenstate at $t=0$ is then

$$
\nu_{i}(t)=\sum_{a} e^{-i E_{a} t} U_{i a} \nu_{a}
$$

If the neutrino masses are not equal, neither are the energies $E_{a}$. This implies that at a subsequent time, $\nu_{i}(t)$ will be a new superposition of the physical eigenstates than this of eq. 5 and that there is a non-zero probability of finding an eigenstate $\nu_{i}{ }^{\prime}$ in a beam which was purely $\nu_{i}$. In this framework, accelerator experiments provide strong bounds on the mixing between $\nu_{e}$ and $\nu_{\mu}$ [5], as well as between $\nu_{\tau}$ and $\nu_{\mu}$ [6].

- Finally, the LEP experiment on the $Z^{0}$ width [7] limits the number of light $\left(m \leq\left(M_{Z^{0}} / 2\right)\right)$ neutrino doublet fields to three. This bound is also restrictive for other fields that may couple to the $Z^{0}$, such as doublet and triplet Majorons.

If the neutrinos are light, neutrino oscillation experiments are the best candidates to measure the small mass differences $\delta m^{2}$ (from $1 \mathrm{eV}^{2}$ down to $10^{-10} \mathrm{eV}^{2}$ ). Moreover, neutrino oscillations may explain the solar neutrino problem. The mechanism of neutrino oscillations is only effective for large neutrino mixing. However, due to the high density conditions encountered in the sun, the oscillation can be amplified by the MSW effect [8]. The data from the solar neutrino experiments can thus be described either by assuming resonant transitions (MSW-effect) or vacuum oscillations. These require the following ranges for masses and mixing angles:

a) The small mixing angle solution for the MSW effect requires

$$
\begin{aligned}
\delta m_{\nu_{e} \nu_{\alpha}}^{2} & \approx(0.6-1.2) \times 10^{-5} \mathrm{eV}^{2} \\
\sin ^{2} 2 \theta_{\alpha e} & \approx(0.6-1.4) \times 10^{-2} .
\end{aligned}
$$

b) Vacuum oscillations can solve the solar neutrino puzzle if

$$
\begin{aligned}
\delta m_{\nu_{e} \nu_{\alpha}}^{2} & \approx(0.5-1.1) \times 10^{-10} \mathrm{eV}^{2} \\
\sin ^{2} 2 \theta_{\alpha e} & \geq 0.75
\end{aligned}
$$

where $\alpha$ is $\mu$ or $\tau$. The most natural solution in unified models is obtained through the MSWmass and mixing angle ranges. This solution in particular requires a light neutrino Majorana mass of the order

$$
m_{\odot} \approx \sqrt{\delta m^{2}} \approx 3.0 \times 10^{-3} \mathrm{eV}
$$


as already given in (6). Such ultra light masses can be generated effectively in GUT's [9] and SUSY - GUT's [10] by the well known 'see-saw' mechanism [11].

In addition, the atmospheric neutrino problem can be resolved, in the presence of a large mixing and a small mass splitting involving the muon neutrino [12]. In this case, bounds from accelerator and reactor disappearance experiments indicate that for $\nu_{e}-\nu_{\mu}$ or $\nu_{\tau}-\nu_{\mu}$ oscillations

$$
\begin{gathered}
\delta m_{\nu_{\alpha} \nu_{\mu}}^{2} \leq 10^{-2} \mathrm{eV}^{2} \\
\sin ^{2} 2 \theta_{\mu \alpha} \geq 0.51-0.6
\end{gathered}
$$

where $\alpha$ stands for $e, \tau$ and in (12) the larger lower limit for $\sin ^{2} 2 \theta_{\mu \alpha}$ refers to $\nu_{\mu}-\nu_{\tau}$ oscillations. Finally, neutrinos are possible candidates for structure formation provided they have a mass of order $O(\mathrm{eV})$. This value can be consistent with the bounds from neutrinoless double beta $\left(\beta \beta_{0 \nu}\right)$ decay. In terms of the neutrino masses and mixing angles, the relevant $\beta \beta_{0 \nu}$ measurable quantity can be written as

$$
\left|<m_{\nu_{e}}>\right|=\left|\sum_{i}^{3}\left(U_{e i}\right)^{2} m_{\nu_{i}} e^{i \lambda_{i}}\right| \leq 1 e V,
$$

where $e^{i \lambda_{i}}$ is the CP-parity of the $i^{t h}$ neutrino, while $U_{e i}$ are the elements of the unitary transformation relating the weak and mass neutrino eigenstates(the masses $m_{\nu_{i}}$ are positive). It appears therefore, that satisfying all the experimental data demands nearly degenerate mass eigenstates $m_{\nu_{i}} \approx m_{0}, i=1,2,3$ 13. Indeed, structure formation in the Universe and the COBE data requires $\sum_{i} m_{i} \approx 3 \mathrm{eV}$, thus setting the scale of the masses. A solution to the atmospheric and solar neutrino deficits, requires the involved masses to be very similar. In this case the $\beta \beta_{0 \nu}$ bound may be respected due to mutual cancellations in (13) by opposite CP-phases $e^{i \lambda_{i}}$.

Passing to neutrino mixing, we see that it may occur either purely from the neutrino sector of the theory, or by the charged lepton mixing. Indeed, in complete analogy to the quark currents the leptonic mixing matrix is $V_{\text {tot }}=V_{\ell} V_{\nu}^{\dagger}$ where $V_{\ell}$ diagonalizes the charged lepton mass matrix, while $V_{\nu}$ diagonalizes the light neutrino mass matrix. In the former case the mixing is typically too small to have any impact on the atmospheric neutrino problem, but may still account for the solar neutrino problem. In the latter case, the mixing may be such as to account for both deficits.

In the simplest scheme with only one large mixing angle, one has two possibilities:

1) The solar neutrino problem is resolved by $\nu_{e}-\nu_{\mu}$ oscillations and the atmospheric neutrino problem by $\nu_{\mu}-\nu_{\tau}$ oscillations. For this possibility to be viable, we need a large mixing angle in the 2-3 entries.

2)The solar neutrino problem is resolved by $\nu_{e}-\nu_{\tau}$ oscillations and the atmospheric neutrino problem by $\nu_{e}-\nu_{\mu}$ oscillations. In this case the large angle should be in the 1-2 entries. 


\section{Derivation of textures from $U(1)$ symmetries}

Extensions of the Standard Model with additional $U(1)$ symmetries can describe the hierarchy of fermion masses and mixing angles, including neutrinos. An example is the model proposed by Ibanez and Ross [14]. The structure of the mass matrices is determined by a family symmetry, $U(1)_{F D}$. The need to preserve $S U(2)_{L}$ invariance requires left-handed up and down quarks (leptons) to have the same charge. This, plus the additional requirement of symmetric matrices, indicates that all quarks (leptons) of the same i-th generation transform with the same charge $\alpha_{i}\left(a_{i}\right)$. The full anomaly free Abelian group involves an additional family independent component, $U(1)_{F I}$, and with this freedom $U(1)_{F D}$ is made traceless without any loss of generality. If the light Higgs, $H_{2}, H_{1}$, responsible for the up and down quark masses respectively, have $U(1)$ charge so that only the $(3,3)$ renormalisable Yukawa coupling to $H_{2}, H_{1}$ is allowed, then only the $(3,3)$ element of the associated mass matrix will be non-zero. The remaining entries are generated when the $U(1)$ symmetry is broken. After this breaking, the mass matrix acquires its structure. Different scales $M_{1}$ and $M_{2}$ are expected for the down and up quark mass matrices, which couple to different Higgs fields. The lepton mass matrix is determined in a similar way and for bottom-tau unification one has the picture

$$
\begin{gathered}
\frac{M_{u}}{m_{t}} \approx\left(\begin{array}{ccc}
\epsilon^{|2+6 a|} & \epsilon^{|3 a|} & \epsilon^{|1+3 a|} \\
\epsilon^{|3 a|} & \epsilon^{2} & \epsilon^{1} \\
\epsilon^{|1+3 a|} & \epsilon^{1} & 1
\end{array}\right), \frac{M_{d}}{m_{b}} \approx\left(\begin{array}{ccc}
\bar{\epsilon}^{|2+6 a|} & \bar{\epsilon}^{|3 a|} & \bar{\epsilon}^{|1+3 a|} \\
\bar{\epsilon}^{|3 a|} & \bar{\epsilon}^{2} & \bar{\epsilon}^{1} \\
\bar{\epsilon}^{|1+3 a|} & \bar{\epsilon}^{1} & 1
\end{array}\right) \\
\frac{M_{\ell}}{m_{\tau}} \approx\left(\begin{array}{ccc}
\bar{\epsilon}^{|2+6 a-2 b|} & \bar{\epsilon}^{|3 a|} & \bar{\epsilon}^{|1+3 a-b|} \\
\bar{\epsilon}^{|3 a|} & \bar{\epsilon}^{|2(1-b)|} & \bar{\epsilon}^{|1-b|} \\
\bar{\epsilon}^{|1+3 a-b|} & \bar{\epsilon}^{|1-b|} & 1
\end{array}\right)
\end{gathered}
$$

The choices $\bar{\epsilon} \equiv\left(\frac{\langle\theta>}{M_{1}}\right)^{\left|\alpha_{2}-\alpha_{1}\right|} \approx 0.23, \epsilon \equiv\left(\frac{\langle\theta>}{M_{2}}\right)^{\left|\alpha_{2}-\alpha_{1}\right|} \approx \bar{\epsilon}^{2}, a=\alpha_{1} /\left(\alpha_{2}-\alpha_{1}\right)=1$ and $\beta \equiv\left(\alpha_{2}-a_{2}\right) /\left(\alpha_{2}-\alpha_{1}\right)=1 / 2,0$ give an excellent phenomenological description.

How are neutrino mass matrices included in such a picture? The first step is to determine the Dirac and heavy Majorana mass matrices. In a scheme where we add three generations of right-handed neutrinos, the light Majorana neutrino mass matrix is then given by

$$
m_{\nu}^{e f f}=\left(m_{\nu}^{D}\right)^{T} \cdot\left(M_{\nu_{R}}^{M}\right)^{-1} \cdot m_{\nu}^{D}
$$

$S U(2)_{L}$ fixes the $U(1)_{F D}$ charge of the left-handed neutrino states to be the same as the charged leptons. The left- right- symmetry then fixes the charges of the right-handed neutrinos to be the same as the left-handed ones. Therefore, the neutrino Dirac mass is [15]

$$
\frac{M_{\nu_{R}}^{D}}{m_{\nu_{\tau}}} \approx\left(\begin{array}{ccc}
\epsilon^{|2+6 a-2 b|} & \epsilon^{|3 a|} & \epsilon^{|1+3 a-b|} \\
\epsilon^{|3 a|} & \epsilon^{|2(1-b)|} & \epsilon^{|1-b|} \\
\epsilon^{|1+3 a-b|} & \epsilon^{|1-b|} & 1
\end{array}\right)
$$

Of course the mass matrix structure of neutrinos is more complicated, due to the possibility of Majorana masses for the right-handed components. These arise from a term of the form 
$\nu_{R} \nu_{R} \Sigma$ where $\Sigma$ is a $S U(3) \otimes S U(2) \otimes U(1)$ invariant Higgs scalar field with $I_{W}=0$ and $\nu_{R}$ is a right-handed neutrino. The possible choices for the $\Sigma U(1)_{F D}$ charge will give a discrete spectrum of possible forms for the Majorana mass, $M_{\nu}^{M}$ [15, 16], whose expansion parameter is the same as the one for the down and lepton mass matrices 15. ¿From this previous work, a single $\Sigma$ field has been used and the main conclusions were the following: (a) in schemes where $\Sigma=\tilde{\bar{\nu}}_{R} \tilde{\bar{\nu}}_{R}$ the heavy Majorana mass scale is typically $10^{13}-10^{14} \mathrm{GeV}$, leading to light neutrinos between $O(4-0.4) \mathrm{eV}$ for a top quark of $O(200) \mathrm{GeV}$ [15]. (b) in a particular scheme [16], the mixing in the $(2,3)$ entries can be quite large. However: (c) in none of the cases does the light Majorana mass matrix have degenerate eigenvalues (d) then, for a single $\Sigma$ field, the structure of the heavy Majorana and Dirac mass matrices results in an even larger mass spread for the light Majorana neutrino masses

However, in principle there is no reason why this particular conclusion in the simplest extension of the Standard Model, should apply in the case of a more complicated symmetry or with more than one pair of singlet fields, $\Sigma, \bar{\Sigma}$ present in the theory [17]. Since in such a case there are many possible patterns, instead of making a complete search based on symmetries, it was easier to work in the opposite way [17], that is: (i) Assume that the neutrino Dirac mass matrix is proportional to the $u$-quark mass matrix. Here, we had looked at the five realistic pairs of texture zero patterns for the quark mass matrices found in 18. For completeness, these appear in table 1 1. (ii) Study all possible Majorana neutrino mass matrices with the maximal number of zeroes, that lead to a large mixing angle and small mass splitting between the neutrinos 2 . (iii) Motivate these phenomenological solutions from symmetries.

\section{Textures for Heavy Majorana mass matrices}

Using the Dirac mass matrices from the previous table, one may look for the structure of the heavy Majorana mass matrices that lead to $m_{\text {eff }}$ with one large angle to solve the atmospheric neutrino. Let us start with an atmospheric neutrino mixing residing in the 2-3 submatrix. The mixing then can be parametrised as

$$
V_{\nu}=\left(\begin{array}{ccc}
1 & 0 & 0 \\
0 & c_{1} & -s_{1} \\
0 & s_{1} & c_{1}
\end{array}\right)
$$

Then $m_{\text {eff }}^{-1}=V_{\nu} m_{e f f}^{-1 d i a g} V_{\nu}^{T}$ will have the form

$$
m_{\text {eff }}^{-1}=\left(\begin{array}{ccc}
\frac{1}{m_{1}} & 0 & 0 \\
0 & \frac{c_{1}^{2}}{m_{2}}+\frac{s_{1}^{2}}{m_{3}} & c_{1} s_{1}\left(\frac{1}{m_{2}}-\frac{1}{m_{3}}\right) \\
0 & c_{1} s_{1}\left(\frac{1}{m_{2}}-\frac{1}{m_{3}}\right) & \frac{c_{1}^{2}}{m_{3}}+\frac{s_{1}^{2}}{m_{2}}
\end{array}\right) \equiv\left(\begin{array}{ccc}
a & 0 & 0 \\
0 & b & d \\
0 & d & c
\end{array}\right) .
$$

where $m_{i}$ are the eigenvalues of $m_{e f f}$. One sees that for $b=c=0$ and $a=d$, three degenerate neutrinos and maximal (2-3) mixing is obtained. Then, the heavy Majorana mass matrix is

\footnotetext{
2 See also [19].
} 


\begin{tabular}{|c|c|c|c|c|}
\hline Solution & $Y_{u}, m_{\nu}^{D}$ & & $Y_{d}$ & \\
\hline \multirow{3}{*}{1} & $\begin{array}{lll}0 & \sqrt{2} \lambda^{6} & 0\end{array}$ & 0 & $2 \lambda^{4}$ & 0 \\
\hline & $\sqrt{2} \lambda^{6} \quad \lambda^{4} \quad 0$ & $2 \lambda^{4}$ & $2 \lambda^{3}$ & $4 \lambda^{3}$ \\
\hline & 0 & 0 & $4 \lambda^{3}$ & 1 \\
\hline \multirow{3}{*}{2} & $\begin{array}{lll}0 & \lambda^{6} & 0\end{array}$ & 0 & $2 \lambda^{4}$ & 0 \\
\hline & $\begin{array}{lll}\lambda^{6} & 0 & \lambda^{2}\end{array}$ & $2 \lambda^{4}$ & $2 \lambda^{3}$ & $2 \lambda^{3}$ \\
\hline & $\left.\begin{array}{lll}0 & \lambda^{2} & 1\end{array}\right)$ & 0 & $2 \lambda^{3}$ & 1 \\
\hline \multirow{3}{*}{3} & $0 \quad \sqrt{2} \lambda^{4}$ & 0 & $2 \lambda^{4}$ & 0 \\
\hline & $\lambda^{4}$ & $2 \lambda^{4}$ & $2 \lambda^{3}$ & $4 \lambda^{3}$ \\
\hline & $\sqrt{2} \lambda^{4} \quad 0$ & 0 & $4 \lambda^{3}$ & 1 \\
\hline \multirow{3}{*}{4} & $\sqrt{2} \lambda^{6} \quad 0$ & 0 & $2 \lambda^{4}$ & 0 \\
\hline & $\sqrt{2} \lambda^{6} \quad \sqrt{3} \lambda^{4} \quad \lambda^{2}$ & $2 \lambda^{4}$ & $2 \lambda^{3}$ & 0 \\
\hline & $\lambda^{2}$ & 0 & 0 & 1 \\
\hline \multirow{3}{*}{5} & $\overline{\lambda^{4}}$ & 0 & $2 \lambda^{4}$ & 0 \\
\hline & $\begin{array}{lll}0 & \sqrt{2} \lambda^{4} & \lambda^{2} / \sqrt{2}\end{array}$ & $2 \lambda^{4}$ & $2 \lambda^{3}$ & 0 \\
\hline & $\lambda^{4} \quad \lambda^{2} / \sqrt{2}$ & 0 & 0 & 1 \\
\hline
\end{tabular}

Table 1: Approximate forms for the symmetric textures.

given by $M_{\nu_{R}}=m_{D}^{\dagger} \cdot m_{\text {eff }}^{-1} \cdot m_{D}$ and can be easily found once we know $m_{D}$. In many unified models, the Dirac neutrino mass is predicted to have the same structure as the up-quark mass. Ramond, Roberts and Ross tabulated the quark textures with the maximal number of zeros, that are consistent with low energy data [18]. Then, we can derive the forms of the heavy Majorana mass matrices that lead to a large mixing, for these solutions [17]. The case of large 2-3 mixing is presented in tables 2 and 3. Textures arising from a large mixing in the 1-2 submatrix appear in table 4 . Here $m_{\text {eff }}^{-1}$ takes a form similar to (17), where the off diagonal elements, $d$, appear in the 1-2 submatrix.

Let us now pass to a discussion of the phenomenology induced by the forms of $m_{\text {eff }}^{-1}$ that have been quoted. We investigate the case of a large mixing in the 2-3 submatrix. The case of large mixing in the 1-2 submatrix is very much the same and leads to analogous conclusions.

There are two possibilities for texture zero solutions: $b=0$ or $c=0$ that follow.

(i) $c=0$

This constraint suggests a rewriting in terms of the parameter $\xi=-\frac{m_{2}}{m_{3}}>0$. Then

$$
c_{1}=\frac{1}{\sqrt{1+\xi}}, \quad s_{1}=\frac{\sqrt{\xi}}{\sqrt{1+\xi}}
$$

\footnotetext{
${ }^{3}$ The case $b=c=0$ e.g. has been already discussed for the Dirac mass matrix pattern 3 of table 1 in section 4 and implies $\xi=1$, is therefore in accordance with (29).
} 


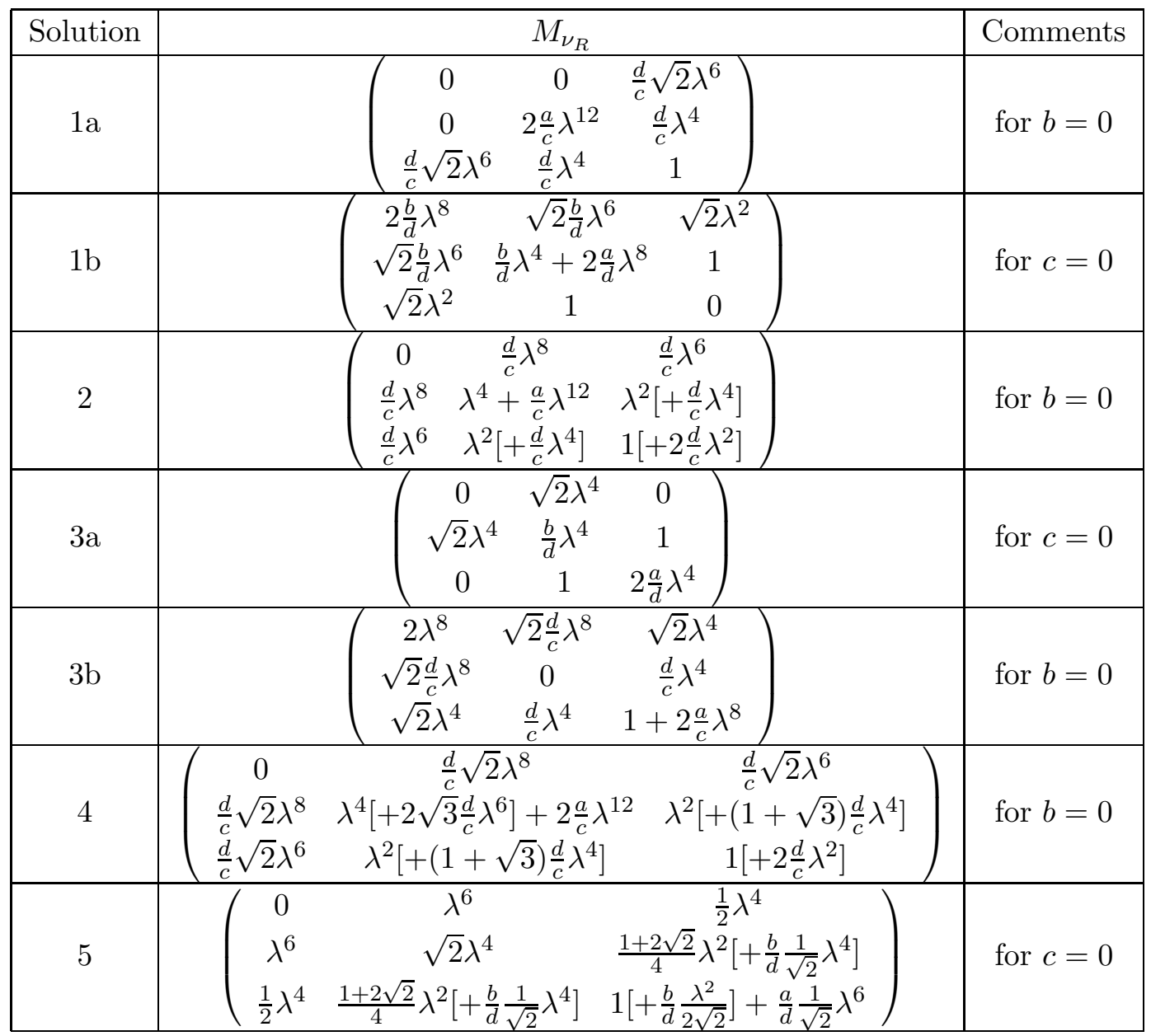

Table 2: The texture zero solutions of the Majorana mass matrices associated with each of the Dirac mass textures of table 1 with a large mixing in the $2-3$ submatrix. We present here cases where either $b=0$ or $c=0$. The non-leading powers are in brackets except for the terms containing the parameter $a=\frac{1}{m_{1}}$. 


\begin{tabular}{|c|c|c|}
\hline Solution & $M_{\nu_{R}}$ & Comments \\
\hline 1 & $\left(\begin{array}{ccc}0 & 0 & \sqrt{2} d \lambda^{6} \\
0 & 2 a \lambda^{12} & d \lambda^{4} \\
\sqrt{2} d \lambda^{6} & d \lambda^{4} & 0\end{array}\right)$ & for $b=c=0$ \\
\hline 2 & $\left(\begin{array}{ccc}0 & d \lambda^{8} & d \lambda^{6} \\
d \lambda^{8} & a \lambda^{12} & d \lambda^{4} \\
d \lambda^{6} & d \lambda^{4} & 2 d \lambda^{2}\end{array}\right)$ & for $b=c=0$ \\
\hline 3 & $\left(\begin{array}{ccc}0 & \sqrt{2} d \lambda^{8} & 0 \\
\sqrt{2} d \lambda^{8} & 0 & d \lambda^{4} \\
0 & d \lambda^{4} & 2 a \lambda^{8}\end{array}\right)$ & for $b=c=0$ \\
\hline 4 & $\begin{array}{ccc}0 & \sqrt{2} d \lambda^{8} & \sqrt{2} d \lambda^{6} \\
\sqrt{2} d \lambda^{8} & 2 \sqrt{3} d \lambda^{6}+2 a \lambda^{12} & d \lambda^{4}+\sqrt{3} d \lambda^{4} \\
\sqrt{2} d \lambda^{6} & d \lambda^{4}+\sqrt{3} d \lambda^{4} & 2 d \lambda^{2}\end{array}$ & for $b=c=0$ \\
\hline 5 & $\left(\begin{array}{ccc}0 & \sqrt{2} d \lambda^{8} & \frac{d \lambda^{6}}{\sqrt{2}} \\
\sqrt{2} d \lambda^{8} & 2 d \lambda^{6} & \frac{d \lambda^{4}}{2}+\sqrt{2} d \lambda^{4} \\
\frac{d \lambda^{6}}{\sqrt{2}} & \frac{d \lambda^{4}}{2}+\sqrt{2} d \lambda^{4} & \sqrt{2} d \lambda^{2}+a \lambda^{8}\end{array}\right.$ & for $b=c=0$ \\
\hline
\end{tabular}

Table 3: Cases as in table 2, but with $b=c=0$.

\begin{tabular}{|c|c|c|}
\hline Solution & $M_{\nu_{R}}$ & Comments \\
\hline 1 & $\left.\begin{array}{ccc}0 & 2 \frac{d}{c} \lambda^{12} & 0 \\
2 \frac{d}{c} \lambda^{12} & 2 \sqrt{2} \frac{d}{c} \lambda^{10}+2 \frac{a}{c} \lambda^{12} & 0 \\
0 & 0 & 1\end{array}\right)$ & for $b=0$ \\
\hline 2 & $\left(\begin{array}{ccc}0 & \frac{d}{c} \lambda^{12} & 0 \\
\frac{d}{c} \lambda^{12} & \lambda^{4}+\frac{a}{c} \lambda^{12} & \lambda^{2}+\frac{d}{c} \lambda^{8} \\
0 & \lambda^{2}+\frac{d}{c} \lambda^{8} & 1\end{array}\right.$ & for $b=0$ \\
\hline 3 & $\left.\begin{array}{ccc}2 \lambda^{8} & 0 & \sqrt{2} \lambda^{4} \\
0 & 0 & \sqrt{2} \frac{d}{c} \lambda^{8} \\
\sqrt{2} \lambda^{4} & \sqrt{2} \frac{d}{c} \lambda^{8} & 1+2 \frac{a}{c} \lambda^{8}\end{array}\right)$ & for $b=0$ \\
\hline 4 & $\begin{array}{ccc}0 & 2 \frac{d}{c} \lambda^{12} & 0 \\
2 \frac{d}{c} \lambda^{12} & 2 \sqrt{6} \frac{d}{c} \lambda^{10}+2 \frac{a}{c} \lambda^{12} & \lambda^{2}+\sqrt{2} \frac{d}{c} \lambda^{8} \\
0 & \lambda^{2}+\sqrt{2} \frac{d}{c} \lambda^{8} & 1\end{array}$ & for $b=0$ \\
\hline 5 & $\left(\begin{array}{ccc}\lambda^{8} & \frac{\lambda^{6}}{\sqrt{2}} & \lambda^{4} \\
\frac{\lambda^{6}}{\sqrt{2}} & \frac{\lambda^{4}}{2} & \frac{\lambda^{2}}{\sqrt{2}}+\sqrt{2} \frac{d}{c} \lambda^{8} \\
\lambda^{4} & \frac{\lambda^{2}}{\sqrt{2}}+\sqrt{2} \frac{d}{c} \lambda^{8} & 1+2 \frac{d}{c} \frac{\lambda^{6}}{\sqrt{2}}+\frac{a}{c} \lambda^{8}\end{array}\right.$ & for $b=0$ \\
\hline
\end{tabular}

Table 4: The texture zero solutions of the Majorana mass matrices associated with each of the Dirac mass textures of table 1 with a large mixing in the 1-2 submatrix, for the examples with $b=0$. Only cases for $b=0$ emerge and the solutions for $a=b=0$ follow immediately. 


$$
m_{\text {eff }}^{-1}=\left(\begin{array}{ccc}
\frac{1}{m_{1}} & 0 & 0 \\
0 & \frac{1-\xi}{m_{2}} & \frac{\sqrt{\xi}}{m_{2}} \\
0 & \frac{\sqrt{\xi}}{m_{2}} & 0
\end{array}\right)
$$

and thus

$$
\sin ^{2} 2 \theta_{1}=\frac{4 \xi}{(1+\xi)^{2}} .
$$

In the neutrino mixing matrix, we add a large angle, as well as a small one, $\theta_{e}$, to explain the solar neutrino problem 1 . The neutrino oscillation probabilities are given in terms of the mixing matrix

$$
\begin{aligned}
V_{t o t}=V_{e}^{\dagger} V_{\nu} & =\left(\begin{array}{ccc}
c_{e} & -s_{e} & 0 \\
s_{e} & c_{e} & 0 \\
0 & 0 & 1
\end{array}\right) \cdot\left(\begin{array}{ccc}
1 & 0 & 0 \\
0 & c_{1} & s_{1} \\
0 & -s_{1} & c_{1}
\end{array}\right) \\
V_{\text {tot }} & =\left(\begin{array}{ccc}
c_{e} & -s_{e} c_{1} & -s_{e} s_{1} \\
s_{e} & c_{e} c_{1} & c_{e} s_{1} \\
0 & -s_{1} & c_{1}
\end{array}\right),
\end{aligned}
$$

where we take

$$
s_{e} \approx \sqrt{\frac{m_{e}}{m_{\mu}}} \approx 0.07, \quad c_{e} \approx 1 .
$$

Such an ansatz for the charged leptons is most commonly used and is suggested by the data. A more general ansatz is definitely more difficult to handle.

It is now straightforward to calculate the oscillations $P\left(\nu_{\alpha} \rightarrow \nu_{\beta}\right)$ :

$$
\begin{gathered}
P\left(\nu_{\mu} \rightarrow \nu_{\tau}\right)=c_{e}^{2} \frac{4 \xi}{(1+\xi)^{2}} \sin ^{2} \frac{m_{2}^{2}\left(1 / \xi^{2}-1\right) x}{4 E_{\nu}} \\
P\left(\nu_{e} \rightarrow \nu_{\tau}\right)=s_{e}^{2} \frac{4 \xi}{(1+\xi)^{2}} \sin ^{2} \frac{m_{2}^{2}\left(1 / \xi^{2}-1\right) x}{4 E_{\nu}} \\
P\left(\nu_{e} \rightarrow \nu_{\mu}\right)=\sin ^{2} 2 \theta_{e}\left[\frac{1}{(1+\xi)} \sin ^{2} \frac{\left(m_{2}^{2}-m_{1}^{2}\right) x}{4 E_{\nu}}\right. \\
\left.+\frac{\xi}{(1+\xi)} \sin ^{2} \frac{\left(m_{3}^{2}-m_{1}^{2}\right) x}{4 E_{\nu}}-\frac{\xi}{(1+\xi)^{2}} \sin ^{2} \frac{\left(m_{3}^{2}-m_{2}^{2}\right) x}{4 E_{\nu}}\right] .
\end{gathered}
$$

(ii) $b=0$

In this case we have

$$
c_{1}=\frac{\sqrt{\xi}}{\sqrt{1+\xi}}, \quad s_{1}=\frac{1}{\sqrt{1+\xi}},
$$

\footnotetext{
${ }^{4}$ Renormalisation group effects cause small deviations from the textures we derive at the GUT scale, thus small mass differences between the neutrinos (needed for solving the solar and atmospheric neutrino problems) are created in a natural way from the above textures.
} 


$$
m_{\text {eff }}^{-1}=\left(\begin{array}{ccc}
\frac{1}{m_{1}} & 0 & 0 \\
0 & 0 & \frac{\sqrt{\xi}}{m_{2}} \\
0 & \frac{\sqrt{\xi}}{m_{2}} & \frac{1-\xi}{m_{2}}
\end{array}\right)
$$

and again the expression (20) for $\sin ^{2} 2 \theta_{1}$. The oscillation probabilities for $\nu_{\mu} \rightarrow \nu_{\tau}$ and $\nu_{e} \rightarrow \nu_{\tau}$ remain the same. For the oscillation $\nu_{e} \rightarrow \nu_{\mu}$ we have to substitute $\xi \rightarrow \frac{1}{\xi}$.

One may now compare these two possibilities for textures with the data. The atmospheric neutrino data implies via (11) that $\xi$ in between

$$
\xi_{1}=0.23 \text { and } \xi_{2}=4.4 \text {. }
$$

Given that $\xi_{1} \xi_{2}=1$, the value of $\xi$ selected merely determines which of the neutrino masses is heavier, as well as the magnitude of the masses. Indeed, from $m_{3}^{2}=\frac{\delta m^{2}}{1-\xi^{2}}$ and $m_{2}^{2}=m_{3}^{2}-\delta m^{2}$, we observe that, for a value $\delta m^{2} \approx 0.01 \mathrm{eV}^{2}$ as implied by the atmospheric neutrino data only values of $\xi$ very near unity would give neutrino masses of order $O(1) \mathrm{eV}$. In particular, one may see that

$$
m_{3} \approx m_{2} \approx 1 \mathrm{eV}, \text { for } \xi=0.995
$$

After accommodating the atmospheric neutrino data, one can turn to the discussion of the solar neutrino numbers, and in this example we interpret them as $\nu_{e} \rightarrow \nu_{\mu}$ oscillations. From (26) we may obtain an effective $\sin ^{2} 2 \theta_{e \mu}$. Depending on the size of $\xi$, the $\frac{1}{1+\xi}$ or $\frac{\xi}{1+\xi}$ term dominates.

$$
\begin{aligned}
& \xi \ll 1: \sin ^{2} 2 \theta_{e \mu} \approx \sin ^{2} 2 \theta_{e} \frac{1}{1+\xi} \approx 1.6 \cdot 10^{-2}, \\
& \xi \gg 1: \sin ^{2} 2 \theta_{e \mu} \approx \sin ^{2} 2 \theta_{e} \frac{\xi}{1+\xi} \approx 1.6 \cdot 10^{-2},
\end{aligned}
$$

when inserting the values of $\theta_{e}$ and $\xi$. This is just in agreement with the MSW solution (7). To satisfy the mass constraints, $m_{1}$ must be nearly equal to $m_{2}$. For an average mass $m_{0} \approx 1$ $\mathrm{eV}, \delta m_{12}^{2} \approx 2 m_{0}\left|m_{2}-m_{1}\right| \approx 10^{-5} \mathrm{eV}^{2}$ indicates the need for a very big degeneracy. Such a high degree of degeneracy is extremely hard to explain from an underlying theory without fine tuning, unless the masses are forced to such values by symmetries, as we are going to discuss shortly.

What about neutrinoless double $\beta$-decay and the COBE data? For the first one, we obtain

$$
\left|<m_{\nu_{e}}>\right|=\left|c_{e}^{2} m_{1}+e^{i\left(\lambda_{2}-\lambda_{1}\right)} s_{e}^{2}\left(c_{1}^{2}-\frac{s_{1}^{2}}{\xi} e^{i\left(\lambda_{3}-\lambda_{2}\right)}\right) m_{2}\right|,
$$

where $e^{i\left(\lambda_{2}-\lambda_{1}\right)}$ is the relative CP eigenvalue of $\nu_{1}$ and $\nu_{2}$ (the masses here are positive). Taking $\nu_{2}$ and $\nu_{3}$ to have the same CP eigenvalues (as already discussed in section 2), we obtain

$$
\left|<m_{\nu_{e}}>\right|=\left|c_{e}^{2} m_{1}+e^{i\left(\lambda_{2}-\lambda_{1}\right)} s_{e}^{2}\left(c_{1}^{2}-\frac{s_{1}^{2}}{\xi}\right) m_{2}\right| .
$$

Now we may again study the texture zeroes. With (18) we get

$$
\left|<m_{\nu_{e}}>\right|=c_{e}^{2} m_{1} \approx m_{1}=O(1) \mathrm{eV}
$$


which is consistent with the bound (13). The above predictions are consistent with the COBE data, as well, since the sum of the masses for the parameter range we indicate, can be of order a few eV's, as required. An identical situation occurs when the large mixing which explains the atmospheric neutrino deficit is in the $1-2$ entries of the neutrino mass matrices.

Let us now see how the quoted mass matrices may arise due to symmetries. The model of [14], which correlates a texture zero in the $(1,3)$ position with a texture zero in the $(1,1)$ position, is consistent with solutions 1, 2 and 4 of [18]. However, the structures 3 and 5 can also arise from realistic flavour symmetries [17, 20]. Indeed, in specific GUT groups, the appearance of zero Clebsch coefficients is to be expected and in [20] we derived the zero texture structures for the Pati-Salam gauge group combined with a $U(1)$ flavour symmetry. The same is true in the presence of residual $Z_{2}$ symmetries, and non-trivial mixing in the Higgs-sector [17]. What about the Heavy Majorana mass matrices? The structures we need require the presence of more than one singlet fields of the $\Sigma$ type; however this is not a problem, as in realistic models many singlets with different $U(1)$ quantum numbers are expected to appear. Let us then give an example of how the heavy Majorana mass matrices that we presented in the tables, may arise from a single $U(1)$ symmetry. Assume the existence of a $\Sigma$ field with a charge -1 (which makes the $(2,3)$ entry unity), as well as the existence of a second field $\Sigma^{\prime}$, with charge +2 . Then, the dominant element in the mass matrix will be the one with the biggest absolute power in $\bar{\epsilon}$. In particular, the elements $(2,2),(2,3)$ and $(3,3)$ still would couple to $\Sigma_{1}$ with charge -1 , while the $(1,2)$ and $(1,3)$ will couple to $\Sigma_{2}$. Then the matrix will be of the form

$$
M_{\nu_{R}} / M_{N}=\left(\begin{array}{ccc}
0 & \bar{\epsilon} & \bar{\epsilon}^{2} \\
\bar{\epsilon} & \bar{\epsilon} & 1 \\
\bar{\epsilon}^{2} & 1 & \bar{\epsilon}
\end{array}\right)
$$

where

$$
M_{N}=m_{t}^{2} d \lambda^{4}=m_{t}^{2} d \bar{\epsilon} / \sqrt{2}
$$

where $m_{t}$ is the top quark mass.

$$
M_{N}=1.5 \times 10^{13}
$$

The structure of the matrix would be that of the example 3a in Table 3. This, as we see, is in fact the solution with only $c=0$ (where the $(2,2)$ element is of order $\bar{\epsilon}$, and $\bar{\epsilon}=\lambda^{4} / \sqrt{2}$ ).

¿From neutrinoless double beta decay one can extract the limit 22]

$$
\left|<1 / M_{N_{e}}>\right|^{-1} \geq 1.6 \times 10^{5}
$$

This arises when the heavy intermediate Majorana neutrinos become dominant.By diagonalizing the matrix $M_{\nu_{R}}$ we find

$$
\left|<1 / M_{N_{e}}>\right|=\left|\sum_{i}^{3}\left(U_{e i}\right)^{2}\left(1 / M_{N_{i}}\right) e^{i \lambda_{i}}\right|
$$

where $M_{N_{i}}$ are the heavy eigenmasses. Using $\bar{\epsilon}=.23$ we find

$$
\left|<1 / M_{N_{e}}>\right|=1 / .0118 M_{N}
$$


which leads to

$$
\left|<1 / M_{N_{e}}>\right|^{-1}=1.6 \times 10^{11}
$$

which is consistent with the above bound and shows that in our model the heavy right handed neutrino contribution is unobservable in neutrinoless double beta decay.

\section{Summary}

It is possible to derive simple Majorana mass matrices of right-handed neutrinos, which may explain the neutrino experimental data (atmospheric neutrino oscillations, solar neutrino oscillations in the MSW approach, neutrinoless double $\beta$-decay and the COBE data). This requires the existence of a right handed neutrino Majorana mass matrix $M_{\nu_{R}}$ with a scale $\left(10^{12}-10^{13}\right)$ $\mathrm{GeV}$. The solution of the atmospheric neutrino puzzle resides in a large mixing stemming from

the neutrino mass matrix. Some type of unification or partial unification implying $m_{\nu}^{D} \sim m_{u}$ was adopted. Along these lines, one can make a classification of heavy Majorana mass matrices, that for a particular type of neutrino (and up-quark) Dirac mass matrix are consistent with the data.

\section{References}

[1] The literature on the subject is vast. Some of the many references are: H. Fritzsch, Phys. Lett. 70B (1977) 436; B73 (1978) 317; Nucl. Phys. B155 (1979) 189; C. D. Froggatt and H. B. Nilsen, Nucl. Phys. B147 (1979) 277; J. Harvey, P. Ramond and D. Reiss, Phys. Lett. B92 (1980) 309; C. Wetterich, Nucl. Phys. B261 (1985) 461; P. Kaus and S. Meshkov, Mod. Phys. Lett. A3 (1988) 1251; F.J. Gilman and Y. Nir, Ann. Rev. Nucl. Part. Sci. \#1 (1990) 213; S. Dimopoulos, L. J. Hall and S. Raby, Phys. Rev. Lett. \#1 (1992) 1984; Phys. Rev. C45 (1992) 4195; H. Arason, D. J. Castaño, P. Ramond and E. J. Piard, Phys. Rev. C47 (1993) 232; G. K. Leontaris and N. D. Tracas, Phys. Lett. B303 (1993) 50; B.C. Allanach and S.F. King, Nucl.Phys. B456 (1995) 57.

[2] Y. Achiman and T. Greiner, Nucl. Phys. B443 (1995) 3; P. Binetruy and P. Ramond, Phys. Lett. B350 (1995) 49; P. Binetruy, S. Lavignac and P. Ramond, Nucl. Phys. B477 (1996) 353; E. Papageorgiu, Z. Phys. C64 (1994) 509; Y. Grossman and Y. Nir, Nucl. Phys. B448 (1995) 30; B.C. Allanach and S.F. King, Nucl.Phys. B459 (1996) 75.

[3] Y. Totsuka, International Symbosium on Lepton Photon Interactions, July 1997, Hamburg.

[4] For a review, see J.D. Vergados, Phys. Rep. 133 (1986) 1; S. M. Bilenky and S. T. Petcov, Rev. Mod. Phys. 59 (1987) 671. Also: R. N. Mohapatra and P. B. Pal, "Massive neutrinos in physics and astrophysics", World Scientific (1991).

[5] L. A. Ahrens et al., Phys. Rev. D 31, 2732 (1985). 
[6] N. Ushida et al., Phys. Rev. Lett. 57, 2897 (1986).

[7] ALEPH Collab., D. Decamp et al., Phys. Lett. B 235, 399 (1990); OPAL Collab., M. Z. Akrawy et al., Phys. Lett. B 240, 497 (1990); DELPHI Collab., P. Abreu et al., Phys. Lett. B 241, 435 (1990); L3 Collab., B. Adeva et al., Phys. Lett. B 249, 341 (1990).

[8] See for example, L. Wolfenstein, Phys.Rev. D17 (1978) 20; S. P. Mikheyev and A. Yu Smirnov, Yad. Fiz. 42 (1985) 1441; J. N. Bahcall and W.C. Haxton, Phys.Rev. D40 (1989) 931; X. Shi, D. N. Schramm and J. N. Bahcall, Phys.Rev.Lett. 69 (1992) 717; P. I. Krastev and S. Petcov, Phys.Lett. B299 (1993) 94; N. Hata and P. Langacker, Phys.Rev. D50 (1994) 632 and references therein.

[9] P. Langacker, Phys. Rept. 72 (1981) 185.

[10] G. G. Ross, Grand Unified Theories, Benjamin Cummings (1985) and references therein.

[11] T. Yanagita, Prog. Theor. Phys. B135 (1978) 66; M. Gell Mann, P. Ramond and R. Slansky, Supergravity (Eds. P. van Nieuwenhuizen, D. Friedmann, 1979).

[12] M. S. Berger et. all, Phys. Lett. B245 (1990) 305; K. S. Hirata et al., Phys. Lett. B280 (1992) 164; R. Becker-Szendy et al., Phys. Rev. D46 (1992) 3720; Y. Fukuda et al., Phys. Lett. B335 (1994) 237.

[13] S. Petcov and A. Smirnov, Phys.Lett. B322 (1994) 109.

[14] L.E. Ibáñez and G.G. Ross, Phys. Lett. B332 (1994) 100.

[15] H. Dreiner, G. K. Leontaris, S. Lola, G. G. Ross and C. Scheich, Nucl. Phys. B346 (1995) 461.

[16] G. Leontaris, S. Lola and G. G. Ross, Nucl. Phys. B454 (1995) 25; G. Leontaris and S. Lola, hep-ph/9510340, $5^{\text {th }}$ Hellenic School and Workshops on Elementary Particle Physics, Corfu, September 1995.

[17] G. Leontaris, S. Lola, C. Scheich and J. Vergados, Phys. Rev. D53 (1996) 6381.

[18] P.Ramond, R.G. Roberts and G.G. Ross, Nucl. Phys. B406 (1993) 19.

[19] P. Binetruy et. al, Nucl. Phys. B496 (1997) 3.

[20] B.C.Allanach, S.F. King, G.K. Leontaris and S. Lola, Phys. Rev. D56 (1997) 2632; Phys. Lett. B407 (1997) 275.

[21] J. Pati and A. Salam, Phys. Rev. D10 (1974) 275.

[22] G.Pantis,F.Simkovic,J.D.Vergados and Amand Faessler, Phys.Rev. C53 (1997) 695. 